\title{
Role of long non-coding RNA HULC in cell proliferation, apoptosis and tumor metastasis of gastric cancer: A clinical and in vitro investigation
}

\author{
YAN ZHAO, QINHAO GUO, JIEJING CHEN, JUN HU, SHUWEI WANG and YUEMING SUN \\ Department of Minimally Invasive Surgery, The First Affiliated Hospital of Nanjing \\ Medical University, Nanjing, Jiangsu 210029, P.R. China
}

Received October 10, 2013; Accepted November 4, 2013

DOI: $10.3892 / o r .2013 .2850$

\begin{abstract}
Long non-coding RNAs (lncRNAs) are emerging as key molecules in human cancer. Highly upregulated in liver cancer (HULC), an lncRNA, has recently been revealed to be involved in hepatocellular carcinoma development and progression. It remains unclear, however, whether HULC plays an oncogenic role in human gastric cancer (GC). In the present study, we demonstrated that HULC was significantly overexpressed in GC cell lines and GC tissues compared with normal controls, and this overexpression was correlated with lymph node metastasis, distant metastasis and advanced tumor node metastasis stages. In addition, a receiver operating characteristic (ROC) curve was constructed to evaluate the diagnostic values and the area under the ROC curve of HULC was up to 0.769. To uncover its functional importance, gain- and lossof-function studies were performed to evaluate the effect of HULC on cell proliferation, apoptosis and invasion in vitro. Overexpression of HULC promoted proliferation and invasion and inhibited cell apoptosis in SGC7901 cells, while knockdown of HULC in SGC7901 cells showed the opposite effect. Mechanistically, we discovered that overexpression of HULC could induce patterns of autophagy in SGC7901 cells; more importantly, autophagy inhibition increased overexpression of HULC cell apoptosis. We also determined that silencing of HULC effectively reversed the epithelial-to-mesenchymal
\end{abstract}

Correspondence to: Professor Yueming Sun, Department of Minimally Invasive Surgery, The First Affiliated Hospital of Nanjing Medical University, 300 Guangzhou Road, Nanjing, Jiangsu 210029, P.R. China

E-mail: jsssunym@sina.com

Abbreviations: IncRNA, long non-coding RNA; GC, gastric cancer; HULC, highly upregulated in liver cancer; AJCC, American Joint Committee on Cancer; EMT, epithelial-to-mesenchymal transition; 3-MA, 3-methyladenine; mRNA, messenger RNA

Key words: autophagy, gastric cancer, long non-coding RNA, epithelial-to-mesenchymal transition, highly upregulated in liver cancer transition (EMT) phenotype. In summary, our results suggest that HULC may play an important role in the growth and tumorigenesis of human GC, which provides us with a new biomarker in GC and perhaps a potential target for GC prevention, diagnosis and therapeutic treatment.

\section{Introduction}

Gastric cancer (GC) is the second leading cause of cancerrelated mortality globally (1). There are $\sim 750,000$ new cases diagnosed annually around the world and 5-year overall survival rates are $<25 \%$ (2). In spite of the progress in understanding the pathophysiological mechanisms and treatment for GC in recent years, the overall survival time of GC patients has not changed significantly. Thus, a deeper understanding of the molecular and genetic networks that control the initiation and progression of GC is imperative.

Long non-coding RNAs (lncRNAs) are a class of newfound non-coding RNAs, <200 nucleotides in length (3). LncRNAs, such as H19, HOTAIR and MEG3, have been suggested to have a functional role in tumorigenesis and tumor progression (4). Highly upregulated in liver cancer (HULC) is $\sim 1.6 \mathrm{k}$ nucleotides long containing two exons but not translated $(5,6)$. It has been shown that HULC might function as a miRNA sponge for miRNA372 and could thereby regulate gene expression at a post-transcriptional level (7). Moreover, Matouk et al (8) reported for the first time that HULC is not HCC-specific and is high in liver nodule of colon cancer origin but not in primary colorectal carcinoma samples and corresponding normal counterparts. However, investigations of HULC in GC are scarce. The expression pattern of HULC in human GC tissues and cell lines, its biological roles, and potential mechanisms in GC progression still need to be addressed.

Epithelial-to-mesenchymal transition (EMT), an essential cell-biological program during embryonic development, contributes to cancer invasion and metastasis $(9,10)$. Autophagy is generally thought to be a double-edged sword in the regulation of tumor progression and some studies showed that autophagy activation is upregulated in cancer cells and contributes to tumor cell survival $(11,12)$. Furthermore, it has been shown that EMT involves the autophagy activation of several important pathways that help tumors survive and 
evolve into highly invasive and metastatic variants (13). In the present study, we examined the role of HULC in GC and the potential mechanisms involved by a retrospective analysis of 58 GC patients, and by carrying out in vitro experiments to clarify the contribution of HULC to various aspects of the malignant phenotype of human GC and its effect on autophagy and EMT.

\section{Materials and methods}

Tissue specimens. Fifty-eight specimens of GC tissues and adjacent non-cancer tissues were surgically obtained between January 2012 and May 2013 at the First Affiliated Hospital of Nanjing Medical University (median age, 64; range, 45-84). Written informed consent was obtained from all patients prior to sample collection. The matched normal gastric tissue samples were obtained from tissues that were $5 \mathrm{~cm}$ from the edge of the tumor and there were no obvious tumor cells, as evaluated by a pathologist. Tissue specimens were immediately frozen in liquid nitrogen after surgery and stored at $-80^{\circ} \mathrm{C}$ until the extraction of total RNA. TNM disease stage was classified according to the American Joint Committee on Cancer (AJCC), 7th Edition. None of the patients recruited in the present study received any preoperative treatments.

Cell lines and cell culture. Human gastric epithelial mucosa cell line GES-1, GC cell lines SGC7901, MKN28, MKN45, AGS and BGC823 were maintained in our laboratory. The cells were cultured in RPMI-1640 containing $10 \%$ fetal bovine serum (FBS) (Gibco, Grand Island, NY, USA), $100 \mathrm{U} / \mathrm{ml}$ of penicillin sodium at $37^{\circ} \mathrm{C}$ in a humidified environment containing $5 \% \mathrm{CO}_{2}$.

Quantitative real-time ( $R T)$-PCR. Total RNA from cells and tissues was extracted using TRIzol reagent (Invitrogen, Carlsbad, CA, USA). cDNA was synthesized using the PrimeScript RT kit (Takara, Dalian, China). Quantitative RT-PCR was performed with FastStart Universal SYBR-Green Master (Rox) (Roche Diagnostics, Indianapolis, IN, USA) with an ABI 7500 (Applied Biosystems, Life Technologies Corporation, Carlsbad, CA, USA). The qPCR cycling was performed as follows: initial denaturation at $95^{\circ} \mathrm{C}$ for $10 \mathrm{~min}$ followed by 40 cycles of denaturation at $95^{\circ} \mathrm{C}$ for $10 \mathrm{sec}$, annealing for $60 \mathrm{sec}$ at $55^{\circ} \mathrm{C}$ (HULC), $50^{\circ} \mathrm{C}$ (E-cadherin) or $60^{\circ} \mathrm{C}$ (vimentin) and finally a melting curve profile was set at $60^{\circ} \mathrm{C}(30 \mathrm{sec})$. Primers for qRT-PCR were synthesized by Invitrogen (Shanghai, China) and the sequences were: HULC sense, 5'-ACTCTGAAGTAAAGGCCGGA-3' and antisense, 5'-TGCCAGGAAACTTCTTGCTTG-3'; E-cadherin sense, 5'-GTGTCATCCAACGGAATGC-3' and antisense, 5'-TGG CGGCATTGTAGGTGTTC-3'; vimentin sense, 5'-ATGAC CGCTTCGCCAACTAC-3' and antisense, 5'-CGGGCTTTG TCGTTGGTTAG-3'. $\beta$-actin was used as an internal control, and the following primer sequences were used to amplify $\beta$-actin: forward, 5'-CTACAATGAGCTGCGTGTGG-3' and reverse, 5'-AAGGAAGGCTGGAAGAGTGC-3'. PCR amplifications were performed in three duplicates for each sample.

Lentivirus packaging and stable transfection cell line generation. To further investigate the function of HULC,
HULC expression was modified by gene knockdown and overexpression via lentivirus vector. We modified the commercial LV-HULC vector and LV-HULC-30 vector lentiviral constructs (Shanghai Genepharma Co., Ltd., Shanghai, China) to overexpress or knock down HULC in GC cells. For knockdown, LV-HULC-30 (target sequence: 5'-GCCTTTACA AGGGAATGAAGA-3'), with $\geq 75 \%$ knockdown efficiency, was used for further studies. All lentiviral vectors expressed GFP and the efficiency of infection was measured under a fluorescent microscope based on GFP expression.

Western blot analysis. Cells were collected and lysed with RIPA lysis buffer (Beyotime Institute of Biotechnology, Shanghai, China). Equal amount of protein $(30 \mu \mathrm{g})$ was loaded and separated on SDS-PAGE, and then transferred to polyvinylidene fluoride membranes (Millipore, Bedford, MA, USA). The membranes were blocked with $5 \%$ non-fat milk in Tris-buffered saline solution containing $0.05 \%$ Tween-20 and then incubated with antibodies specific for E-cadherin, vimentin, LC3-I and LC3-II (1:5,000; Cell Signaling Technology). Following incubation with goat anti-rabbit IgG (1:1,000; Cell Signaling Technology) at $37^{\circ} \mathrm{C}$ for $2 \mathrm{~h}$, bound proteins were visualized using ECL (Pierce) and detected using a Bio-Imaging System. Protein levels were normalized to GAPDH (1:10,000; Cell Signaling Technology) and changes were determined.

Cell proliferation assay. Cell proliferation was evaluated using the Cell Counting Kit-8 (CCK-8; Beyotime Institute of Biotechnology) and following the manufacturer's instructions. Briefly, cells were plated in 96-well plates in medium containing $10 \%$ FBS at $\sim 5 \times 10^{3}$ cells/well. Then $10 \mu \mathrm{l} \mathrm{CCK-8}$ solution was added to each well and incubated for $1 \mathrm{~h}$. The absorbance at $450 \mathrm{~nm}$ was measured using a microplate reader. Results are representative of three individual experiments in triplicate.

Wound healing assay. Cells $\left(5 \times 10^{5}\right)$ were seeded in 6-well plates and cultured in complete medium. After $24 \mathrm{~h}$, when the cells were grown to $90-100 \%$ confluency, a single wound was created in the center of the well by removing the attached cells with a sterile $200 \mu \mathrm{l}$ pipette tip. After $24 \mathrm{~h}$ of culturing, the cells which migrated into the wounded area were visualized and photographed under an inverted microscope. Each experiment was performed at least three times independently.

Transwell migration and invasion assay. Transwell invasion assay was performed using Boyden's chambers. Cells were planted in the upper chamber consisting of 8-mm membrane filter inserts coated with Matrigel (BD Biosciences). The chemoattractant in the lower chamber was supplemented with medium containing $10 \%$ FBS. Cells on the upper surface were removed by a wet cotton swab after $24 \mathrm{~h}$, and those attached on the lower side of the membrane were fixed and stained with crystal violet before counting under a microscope in five randomly selected fields. Migration assays were performed the same way as the invasion assays, using Transwell compartment, with the exception that Matrigel was not included. At least three chambers from three different experiments were analyzed. 
A

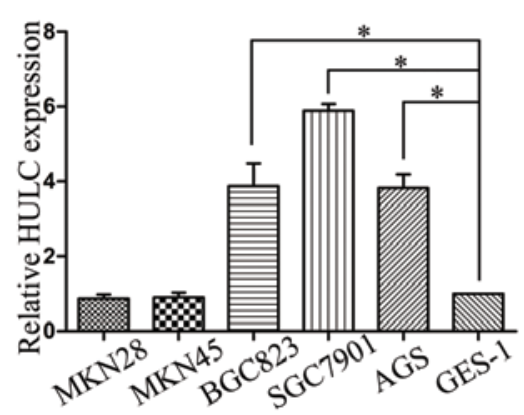

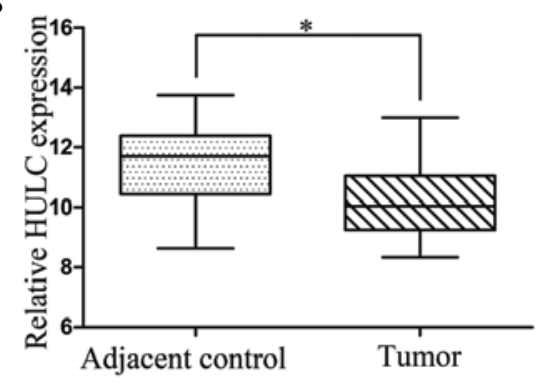

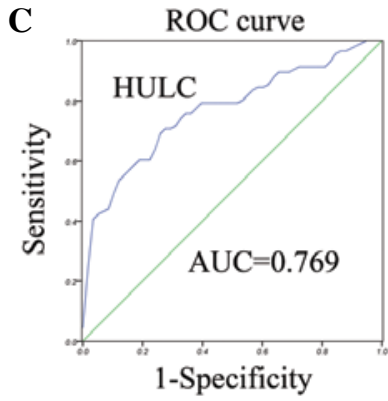

Figure 1. HULC expression and its clinical significance. (A) The relative expression of HULC was measured in various cell lines, including one immortalized gastric cell line (GES-1) and 5 GC cell lines (MKN28, MKN45, SGC7901, AGS and BGC823). The relative HULC level was calculated by $2^{-\Delta \Delta C T}$ where $\Delta \mathrm{C}_{\mathrm{T}}=$ $\mathrm{C}_{\mathrm{T}}$ (HULC) - $\mathrm{C}_{\mathrm{T}}$ ( $\beta$-actin) and $\Delta \Delta \mathrm{C}_{\mathrm{T}}=\Delta \mathrm{C}_{\mathrm{T}}$ (GC cell)- $\Delta \mathrm{C}_{\mathrm{T}}$ (GES-1). (B) Analysis of HULC expression level was performed in GC tissues or adjacent normal tissues. The $\Delta \mathrm{C}_{\mathrm{T}}$ value was determined by subtracting the $\beta$-actin $\mathrm{C}_{\mathrm{T}}$ value from the HULC $\mathrm{C}_{\mathrm{T}}$ value. Smaller $\Delta \mathrm{C}_{\mathrm{T}}$ value indicates higher expression. $(\mathrm{C}) \mathrm{ROC}$ analysis of HULC expression in predicting the aggravation of GC. ${ }^{*} \mathrm{P}<0.05$.

Flow cytometric analysis of apoptosis and autophagy. Cells $(7,000,000)$ treated with LV-HULC or LV-NC-2 or $5 \mathrm{mM}$ 3-methyladenine (3-MA; Sigma) were seeded into 6-cm tissue culture dishes for $24 \mathrm{~h}$. For detection of apoptosis, adherent cells were both collected and resuspended in cold PBS for analysis. Apoptosis was detected using the Alexa Fluor ${ }^{\circledR}$ 647/7-AAD apoptosis kit (BioLegend, San Diego, CA, USA) according to the manufacturer's instructions. Data were assessed by flow cytometry (Becton-Dickinson, San Jose, CA, USA).

Statistical analysis. Statistical Program for Social Sciences (SPSS) 20.0 software (IBM, SPSS, Inc., Chicago, IL, USA) was used for the statistical analysis. Data are expressed as mean \pm standard deviation (SD) from at least three separate experiments. Statistical analyses were performed with the Student's t-test. ROC curve was established to evaluate the diagnostic value for differentiating between GC tissues and normal tissues. Differences were considered to be statistically significant at $\mathrm{P}<0.05$.

\section{Results}

HULC is upregulated in GC tissues and cell lines. To assess the role of HULC in GC progression, we first examined HULC expression levels in the GC cell lines and the human gastric epithelial mucosa cell line GES-1 using qRT-PCR. As presented in Fig. 1A, the expression of HULC was increased in three GC cell lines (SGC7901, BGC823 and AGS) relative to the expression in the human gastric epithelial mucosa cell line GES-1, but there was no significant difference for MKN28 and MKN45. Next, qRT-PCR assays were further developed to quantify HULC in 58 pairs of GC tissues and pair-matched adjacent normal tissues. HULC levels were markedly upregulated in cancerous tissues compared with noncancerous tissues (Fig. 1B). Then, we verified that expression of HULC was significantly correlated with lymph node metastasis, distant metastasis and TNM stages. However, we did not find any association between HULC expression levels and other clinicopathological features including age, gender, differentiation, tumor size, tumor location and CEA values (Table I). Finally, we examined whether HULC could be used as a marker of GC. We used corresponding adjacent non-tumorous tissues as a control to produce a ROC curve. The cut-off value was 10.88 .
Table I. The relationship between HULC expression levels $\left(\Delta \mathrm{C}_{\mathrm{T}}\right)$ and clinicopathological factors of $58 \mathrm{GC}$ patients.

\begin{tabular}{|c|c|c|c|}
\hline Characteristics & $\begin{array}{c}\text { No. of } \\
\text { patients }(\%)\end{array}$ & Mean \pm SD & P-value \\
\hline \multicolumn{4}{|l|}{ Age (years) } \\
\hline$\geq 60$ & $32(55.17)$ & $10.11 \pm 1.08$ & 0.87 \\
\hline$<60$ & $26(44.83)$ & $10.16 \pm 1.83$ & \\
\hline \multicolumn{4}{|l|}{ Gender } \\
\hline Male & $44(75.86)$ & $10.26 \pm 1.16$ & 0.12 \\
\hline Female & $14(24.14)$ & $9.73 \pm 0.86$ & \\
\hline \multicolumn{4}{|l|}{ Diameter $(\mathrm{cm})$} \\
\hline$\geq 5$ (large) & $35(60.34)$ & $10.09 \pm 1.01$ & 0.7 \\
\hline$<5$ (small) & $23(39.66)$ & $10.21 \pm 1.27$ & \\
\hline \multicolumn{4}{|l|}{ Location } \\
\hline Cardia or body & $22(37.93)$ & $10.10 \pm 1.12$ & 0.83 \\
\hline Antrum & $36(62.07)$ & $10.16 \pm 1.13$ & \\
\hline \multicolumn{4}{|l|}{ Differentiation } \\
\hline Poor or not & $34(58.62)$ & $9.98 \pm 1.07$ & 0.20 \\
\hline Well or moderate & $24(41.38)$ & $10.36 \pm 1.16$ & \\
\hline \multicolumn{4}{|c|}{ Lymphatic metastasis } \\
\hline Present & $41(70.69)$ & $9.83 \pm 1.09$ & 0.001 \\
\hline Absent & $17(29.31)$ & $10.87 \pm 0.82$ & \\
\hline \multicolumn{4}{|l|}{ Distal metastasis } \\
\hline Present & $6(10.34)$ & $9.06 \pm 0.46$ & 0.01 \\
\hline Absent & $52(89.66)$ & $10.26 \pm 1.10$ & \\
\hline \multicolumn{4}{|l|}{ AJCC clinical stage } \\
\hline $\mathrm{I}+\mathrm{II}$ & $20(34.48)$ & $10.73 \pm 0.91$ & 0.003 \\
\hline III + IV & $38(65.52)$ & $9.83 \pm 1.10$ & \\
\hline \multicolumn{4}{|l|}{ Serum CEA value } \\
\hline$\geq 5 \mu \mathrm{g} / 1$ & $38(65.52)$ & $10.13 \pm 1.14$ & 0.95 \\
\hline$<5 \mu \mathrm{g} / 1$ & $20(34.48)$ & $10.15 \pm 1.09$ & \\
\hline
\end{tabular}

The area under the ROC curve (AUC) was $0.769(\mathrm{P}<0.001)$. The sensitivity and specificity was 0.707 and 0.724 . The Youden index was 0.431 (Fig. 1C). 
A

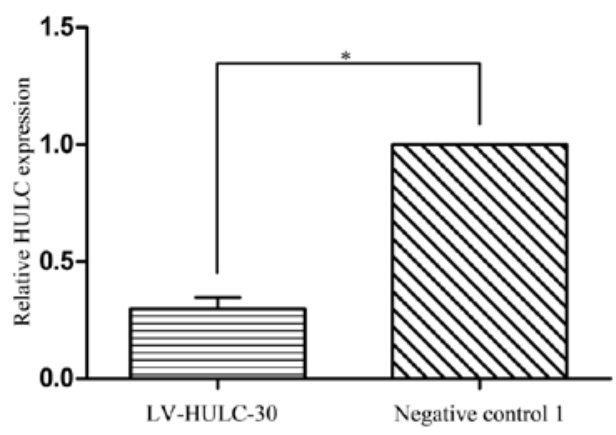

C

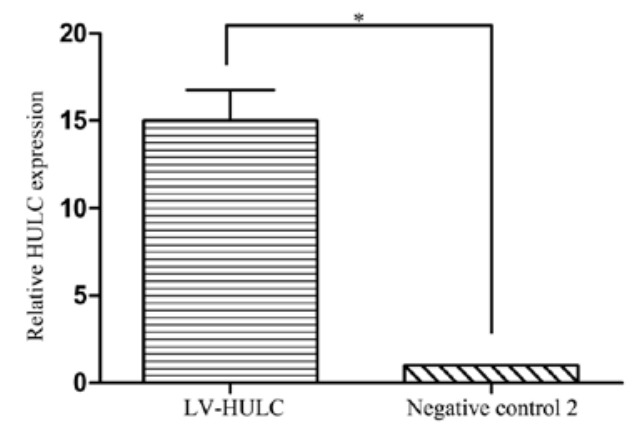

B

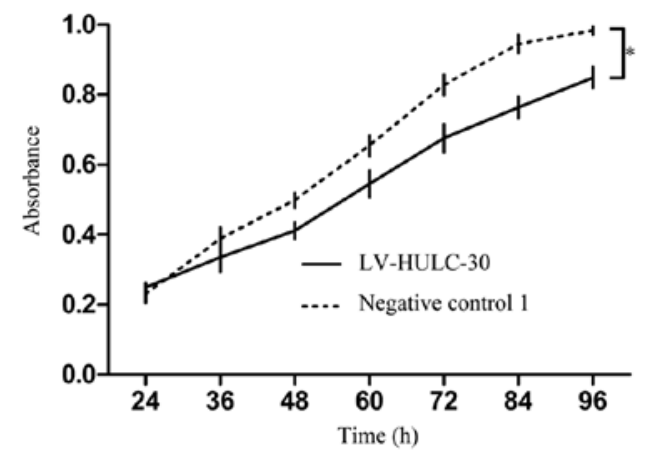

D

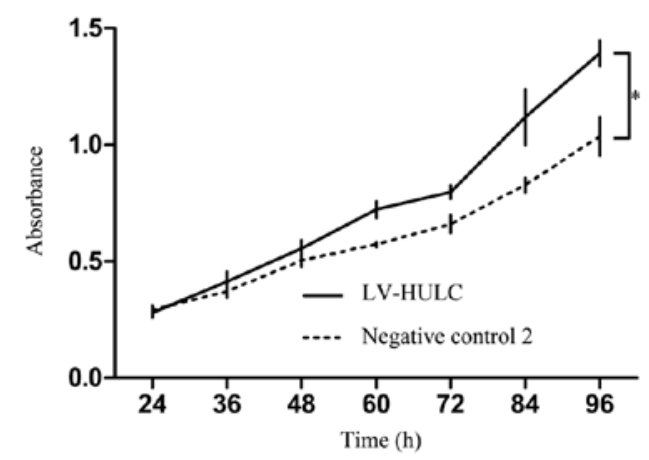

Figure 2. The role of HULC in the regulation of cell proliferation. (A) Knockdown of HULC expression in SGC7901 cells by LV-HULC-30 was confirmed by qRT-PCR. (B) Significant differences were observed between the negative control 1 and HULC-depleted cells. (C) LV-HULC was transfected into SGC7901 cells, and cell lines stably expressed with HULC were screened. HULC levels were significantly increased in SGC7901. (D) Significant differences were observed between the negative control 2 and LV-HULC-transfected SGC7901. ${ }^{*} \mathrm{P}<0.05$.

Abnormally expressed HULC promotes the proliferation of $G C$ cells. To characterize the functional importance of HULC in GC tumorigenesis, we infected SGC7901 cells with either HULC overexpression vector (LV-HULC) or lentivirusmediated HULC silencing vector (LV-HULC-30) to increase or knock down the HULC expression, respectively. qRT-PCR was performed to examine the mRNA levels of HULC in the derived cells (Fig. 2A and C). CCK-8 assays indicated that enhanced expression of HULC promoted cell proliferation in SGC7901 cells. The inhibition of HULC, on the other hand, inhibited cell proliferation (Fig. 2B and D). These results suggest that HULC plays an important role in regulating cell proliferation.

Knockdown of HULC suppresses cell invasion and reverses $E M T$ in GC. Studies indicated that knockdown of HULC inhibited the proliferation of SGC7901. We subsequently investigated the migration and invasion of SGC7901 cells following LV-HULC-30 transfection. The effect of HULC on the migration of SGC7901 cells determined by wound healing assay demonstrated that knockdown of HULC significantly inhibited the migration of SGC7901 cells compared with the SGC7901 only or negative control 1 vector-transfected cells (Fig. 3A). In analogical results observed in the Transwell assay, in comparison with original SGC7901 and negative control cells, the LV-HULC-30 cells showed decreased migration and invasion ability (Fig. 3B-D).

To further define the role of HULC in the progression of cell metastasis in GC cells, we then transfected the LV-HULC-30 vector and assessed the expression of EMT markers at mRNA and protein levels. As shown in Fig. 3E, silencing of HULC in SGC7901 cells induced round spheroids with no or few protrusions. Depletion of HULC expression upregulated E-cadherin and downregulated vimentin expressions at mRNA and protein levels, respectively (Fig. 3F and G). Our in vitro study confirmed that HULC positively regulates GC cell migration and invasion and deletion of HULC reverses EMT, indicating that HULC could act as a possible regulator of EMT.

Upregulated HULC inhibits cell apoptosis by activating autophagy. To explore the mechanisms of HULC in regulating tumorigenesis, we then investigated whether HULC regulates cell apoptosis-related signals, focusing on autophagy. For that purpose, we initially determined a change in the expression level of the microtubule-associated protein 1 light chain 3 (LC3)-II, a marker for the presence of autophagosomes. As shown in Fig. 4A, HULC overexpression resulted in an increase in the ratio of LC3-II/LC3-I. These data suggest that upregulated HULC contributes to autophagy activation in SGC7901. Subsequently, to further study the role of HULC in the regulation of cell apoptosis, SGC7901 cells were treated with LV-HULC or 3-MA (an inhibitor of autophagic sequestration blocker). As depicted in Fig. 4B and C, enhanced expression of HULC inhibited SGC7901 cell apoptosis, whereas autophagy inhibition increased cell apoptosis in SGC7901 cells treated with LV-HULC. 
A
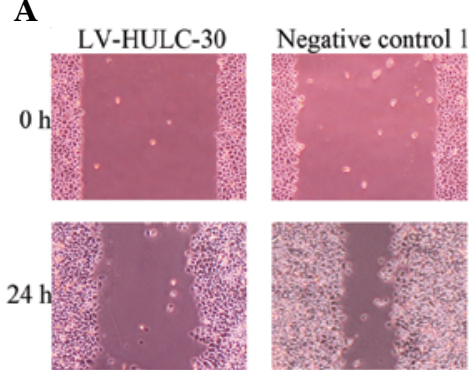

C
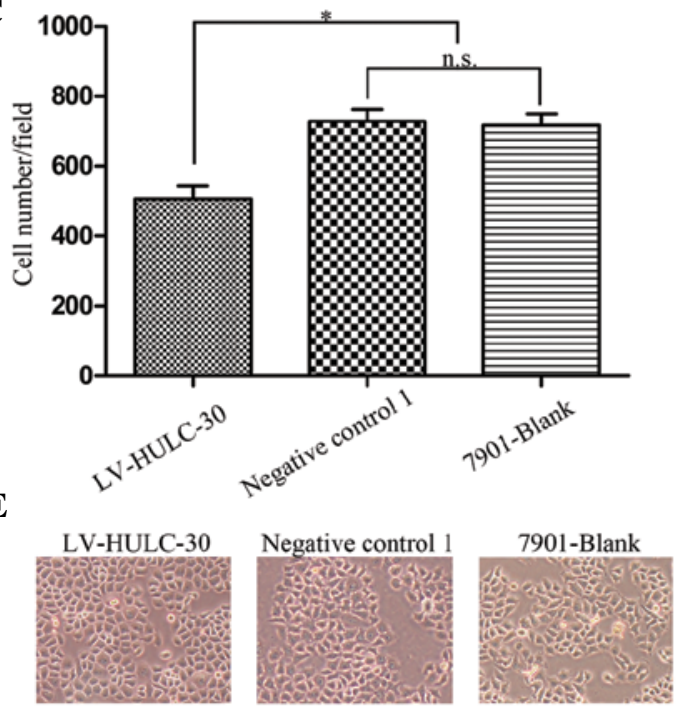

G
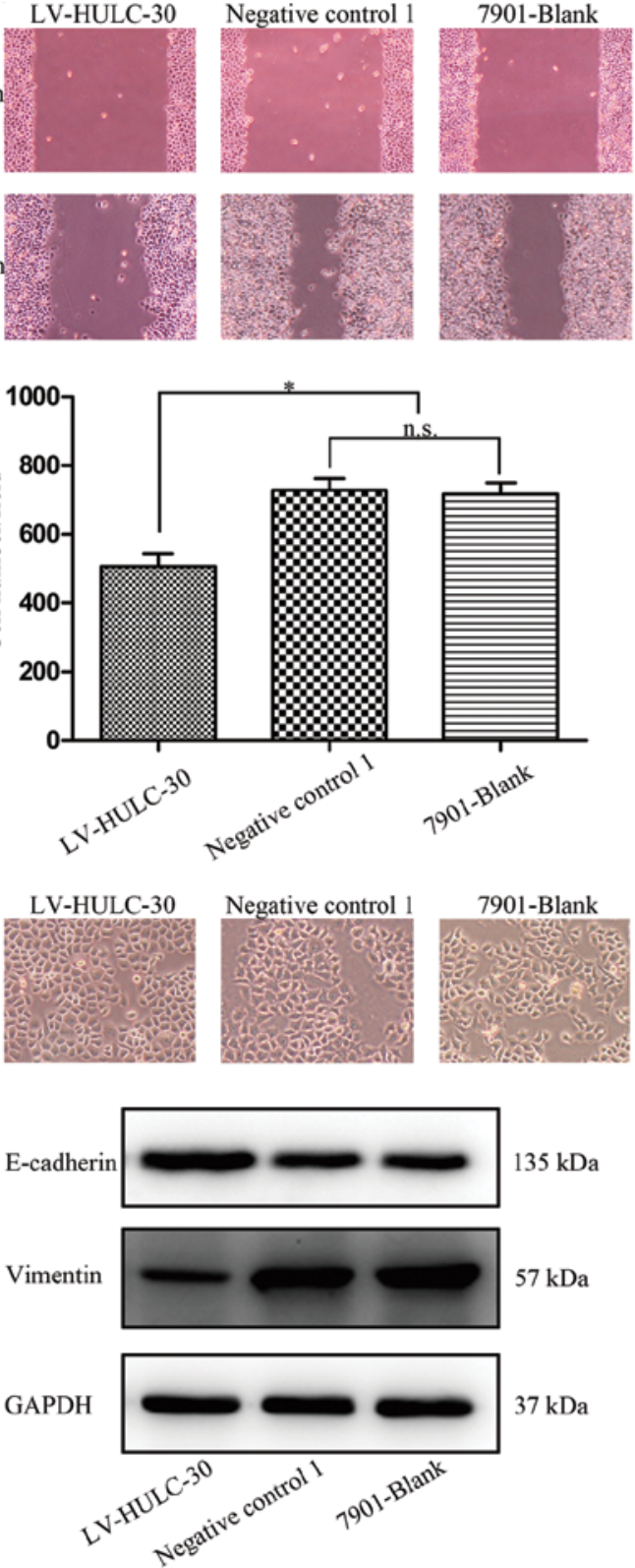

B
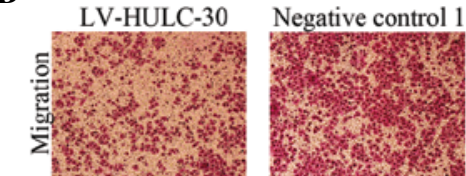

7901-Blank
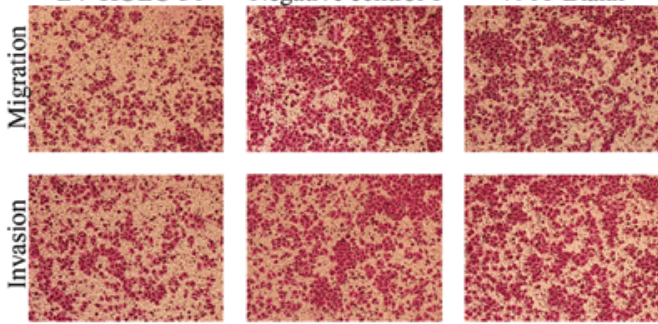

D
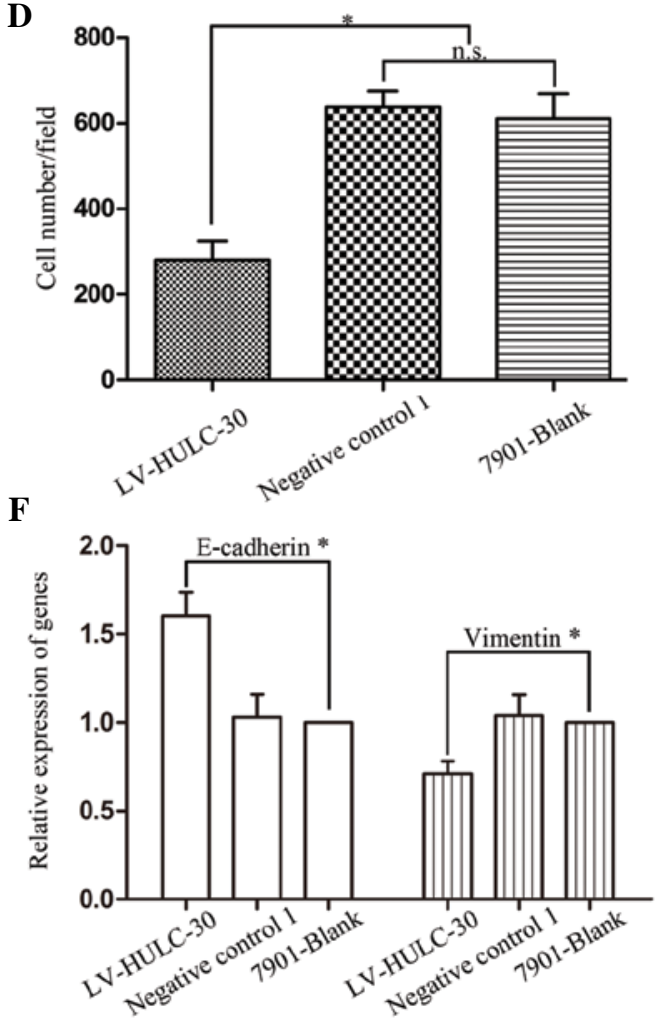

Figure 3. Silencing of HULC suppresses the migratory capacity of SGC7901 cells. (A) Scratch wound-healing assay was conducted in LV-HULC-30-transfected SGC7901 cells and control cells. Migration distance was measured at 0 and $24 \mathrm{~h}$ after cells were scratched. Magnification, x40. (B) Representative images of migrating or invading cells. Magnification, x100. Average number of (C) migrating or (D) invading cells from three independent experiments. (E) Phasecontrast photomicrographs. Downregulation of HULC expression induced morphological changes, such as the inversion of the EMT. Magnification, x100. (F) Analyses of E-cadherin and vimentin mRNA levels were performed in SGC7901 cells after HULC knockdown. (G) Western blot analysis tested the expression level of EMT markers such as E-cadherin and vimentin in SGC7901 cells after HULC knockdown. *P<0.05; n.s., not significant.

\section{Discussion}

Emerging evidence suggests that IncRNAs play a crucial role in the modulation of tumor behavior through various complex mechanisms such as modulating gene transcription and epigenetic signaling; however, limited data are available on the expression and function of IncRNAs in GC (14-17). Hence, in the present study, we tested the expression of a novel IncRNA HULC in GC tissues and cell lines. We also identified the function of HULC in GC cells by applying gain- and lossof-function approaches. Our results demonstrated that HULC is upregulated in GC tissues in comparison with the adjacent normal gastric tissues. Of note, its level was significantly associated with lymph node metastasis, distant metastasis and TNM stages. Alteration of HULC expression provided further functional evidence, supporting the stimulatory effect of HULC on GC via enhancing aggressive biological behavior of cancer cells. There were certain limitations in the present study; all the patients enrolled were from a single institution and the number of samples was not sufficient to make subgroup analysis. For example, only six patients were at M1 status. In future studies, we will expand the samples for further investigation and seek to elucidate the association between HULC expression and overall survival of GC patients since the present study was limited to patients who underwent complete resection and the follow-up period after surgery was short. 
A
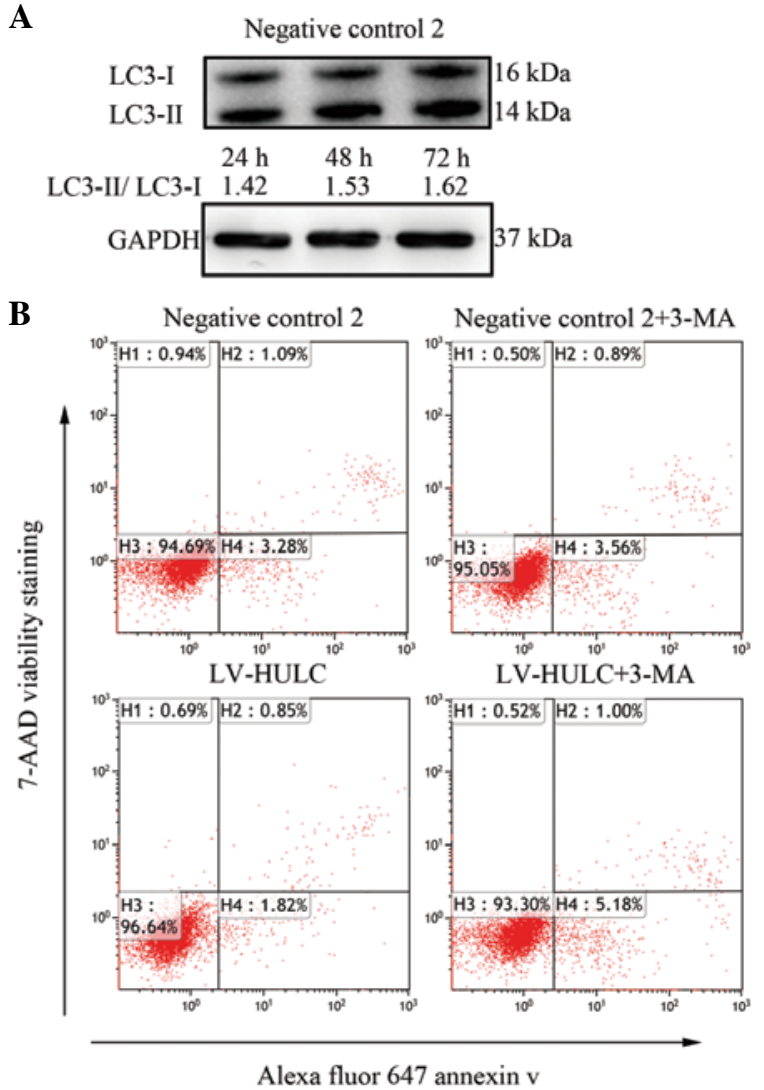

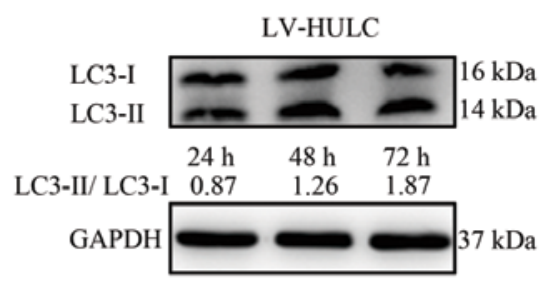

C

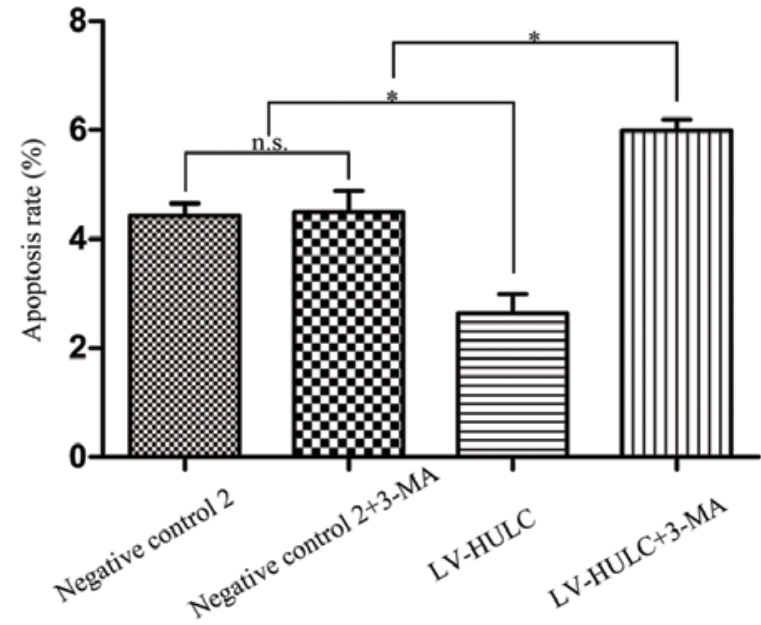

Figure 4. Association between HULC and autophagy in SGC7901 cells. A representative of three independent experiments is shown. (A) SGC7901 cells were treated with LV-HULC or negative control 2, and western blot analysis of LC3-I and LC3-II proteins was performed at 24, 48 and 72 h. The ratio of LC3-II/ LC3-I relative to GAPDH density is reported in the figure as direct marker of autophagy. (B and C) SGC7901 cells were treated with LV-HULC or LV-HULC plus $5 \mathrm{mM} 3$-MA, and apoptosis was detected using flow cytometry. ${ }^{*} \mathrm{P}<0.05$; n.s., not significant.

Metastasis is a complex and multistep process, which has to be divided into 2 phases; namely, physical translocation of a tumor cell from the primary tumor to a distant tissue to seed and colonization of disseminated tumor cell in the tissue (18). A crucial mechanism by which carcinoma cells enhance their invasive capacity is the dissolution of intercellular adhesions and the acquisition of a more motile mesenchymal phenotype as part of an epithelial-to-mesenchymal transition (EMT) (19). During EMT, epithelial markers that are downregulated during this process include E-cadherin, ZO-1 and MUC1 $(20,21)$. Molecules that are upregulated in this process include the transcription factors Snail, Slug, Twist as well as N-cadherin and vimentin $(22,23)$. To determine whether these tumor characteristics initiated by HULC are associated with the EMT, we focused on hallmarks of the EMT phenotype. Notably, in the present study, we showed for the first time that HULC is a positive regulator of EMT. This conclusion was based on the observation that silencing HULC induced a repertoire of biochemical (increased E-cadherin and decreased vimentin) and morphological (growth pattern, decreased formation of lamellipodia) changes that reverse EMT. Furthermore, we confirmed that downregulation of HULC expression decreased the invasion and migration ability of SGC7901 cells. These results indicated that alterative expression of HULC, at least in part, had an impact on the process of EMT by regulating the expression of E-cadherin and vimentin in GC cells, suggesting that HULC plays a causative role in epithelial characteristics weakening. However, the concrete mechanisms of how HULC regulates E-cadherin and vimentin expression in SGC7901 cells remain to be clarified in our future studies

Programmed cell death (PCD), including two classical forms, apoptosis and autophagy, is a crucial mechanism for maintaining cell homeostasis of multicellular organisms. Autophagy, termed type II PCD, is generally activated by conditions of nutrient deprivation, but it has also been associated with a number of physiological processes including development, differentiation, infection and cancer (24-27). However, in cancer development, autophagy has been shown to have a dual role. In some cases, autophagy is a tumor-suppressive mechanism, but in others, autophagy promotes tumorigenesis (28-30). Therefore, one question that has emerged from our studies is whether autophagy displays a cytotoxic or cytoprotective role. Autophagosome marker light chain 3 (LC3) was originally identified as a subunit of microtubule-associated proteins $1 \mathrm{~A}$ and $1 \mathrm{~B}$ and was subsequently found to be similar to the yeast protein Apg8/Aut7/Cvt5 critical for autophagy $(31,32)$. The conversion of LC3 to the lower migrating form, LC3-II, has been used as an indicator of autophagy (33). Studies are beginning to elucidate the association between autophagy and lncRNA. Ying et al (34) demonstrated that downregulated MEG3 promotes cell proliferation by activating autophagy in bladder cancer. In the present study, we provided a line of evidence that HULC may be involved in a molecular switch mechanism between apoptosis and autophagy in SGC7901 
cells. However, despite data from the present study, several issues remain to be clarified, such as the relationship between autophagy and apoptosis, especially in cancer cells, the role of PI3K-Akt-mTOR signaling pathway in autophagy, and the parameters that determine whether autophagy is pro-survival or pro-death, tumor promoting or tumor suppressive. Future studies are required to better understand the function of this novel gene and its role in GC tumorigenesis in vivo.

In the present study, for the first time, we found that high expression levels of HULC, a cancer-related lncRNA, correlated clinically with GC progression. Additionally, HULC contributed to the malignant phenotype of GC cells through its regulation of diverse cellular processes, including proliferation, apoptosis, migration and invasion. Therefore, the above findings not only suggest a useful candidate molecular marker for GC and an indicator for advanced-stage GC, but they also provide new insights into the role of IncRNA in cancer biology.

\section{Acknowledgements}

The present study was supported by the Department of Health of the Jiangsu Province Fund.

\section{References}

1. Konishi H, Ichikawa D, Komatsu S, et al: Detection of gastric cancer-associated microRNAs on microRNA microarray comparing pre- and post-operative plasma. Br J Cancer 106: 740-747, 2012.

2. Cheng Y, Jin Z, Agarwal R, et al: LARP7 is a potential tumor suppressor gene in gastric cancer. Lab Invest 92: 1013-1019, 2012.

3. Costa FF: Non-coding RNAs: new players in eukaryotic biology. Gene 357: 83-94, 2005.

4. Batista PJ and Chang HY: Long noncoding RNAs: cellular address codes in development and disease. Cell 152: 1298-1307, 2013.

5. Panzitt K, Tschernatsch MM, Guelly C, et al: Characterization of HULC, a novel gene with striking up-regulation in hepatocellular carcinoma, as noncoding RNA. Gastroenterology 132: 330-342, 2007.

6. Xu D, Yang F, Yuan JH, et al: Long noncoding RNAs associated with liver regeneration 1 accelerates hepatocyte proliferation during liver regeneration by activating Wnt/ $\beta$-catenin signaling. Hepatology 58: 739-751, 2013.

7. Wang J, Liu X, Wu H, et al: CREB up-regulates long non-coding RNA, HULC expression through interaction with microRNA372 in liver cancer. Nucleic Acids Res 38: 5366-5383, 2010.

8. Matouk IJ, Abbasi I, Hochberg A, Galun E, Dweik H and Akkawi M: Highly upregulated in liver cancer noncoding RNA is overexpressed in hepatic colorectal metastasis. Eur J Gastroenterol Hepatol 21: 688-692, 2009.

9. Li J, Yang B, Zhou Q, et al: Autophagy promotes hepatocellular carcinoma cell invasion through activation of epithelial-mesenchymal transition. Carcinogenesis 34: 1343-1351, 2013.

10. Ledford H: Cancer theory faces doubts. Nature 472: 273, 2011.

11. Enomoto M, Tsuchida A, Miyazawa K, et al: Vitamin K2-induced cell growth inhibition via autophagy formation in cholangiocellular carcinoma cell lines. Int J Mol Med 20: 801-808, 2007.

12. Lv Q, Hua F and Hu ZW: DEDD, a novel tumor repressor, reverses epithelial-mesenchymal transition by activating selective autophagy. Autophagy 8: 1675-1676, 2012.
13. Akalay I, Janji B, Hasmim M, et al: Epithelial-to-mesenchymal transition and autophagy induction in breast carcinoma promote escape from T-cell-mediated lysis. Cancer Res 73: 2418-2427, 2013.

14. Nie Y, Liu X, Qu S, Song E, Zou H and Gong C: Long noncoding RNA HOTAIR is an independent prognostic marker for nasopharyngeal carcinoma progression and survival. Cancer Sci 104: 458-464, 2013.

15. Yang F, Bi J, Xue X, et al: Up-regulated long non-coding RNA H19 contributes to proliferation of gastric cancer cells. FEBS J 279: 3159-3165, 2012.

16. Yang $\mathrm{H}$, Zhong Y, Xie H, et al: Induction of the liver cancerdown-regulated long noncoding RNA uc002mbe. 2 mediates trichostatin-induced apoptosis of liver cancer cells. Biochem Pharmacol 85: 1761-1769, 2013.

17. Wahlestedt C: Targeting long non-coding RNA to therapeutically upregulate gene expression. Nat Rev Drug Discov 12: 433-446, 2013.

18. Liu Q, Huang J, Zhou N, et al: LncRNA loc285194 is a p53-regulated tumor suppressor. Nucleic Acids Res 41: 4976-4987, 2013.

19. Lv Q, Wang W, Xue J, et al: DEDD interacts with PI3KC3 to activate autophagy and attenuate epithelial-mesenchymal transition in human breast cancer. Cancer Res 72: 3238-3250, 2012.

20. Shin NR, Jeong EH, Choi CI, et al: Overexpression of Snail is associated with lymph node metastasis and poor prognosis in patients with gastric cancer. BMC Cancer 12: 521, 2012.

21. Lin X, Shang X, Manorek G and Howell SB: Regulation of the epithelial-mesenchymal transition by claudin-3 and claudin- 4 . PLoS One 8: e67496, 2013.

22. Davidson B, Trope CG and Reich R: Epithelial-mesenchymal transition in ovarian carcinoma. Front Oncol 2: 33, 2012.

23. Zeisberg M and Neilson EG: Biomarkers for epithelial-mesenchymal transitions. J Clin Invest 119: 1429-1437, 2009.

24. Kalluri R and Weinberg RA: The basics of epithelial-mesenchymal transition. J Clin Invest 119: 1420-1428, 2009.

25. Kondo Y, Kanzawa T, Sawaya R and Kondo S: The role of autophagy in cancer development and response to therapy. Nat Rev Cancer 5: 726-734, 2005.

26. Levine B and Yuan J: Autophagy in cell death: an innocent convict? J Clin Invest 115: 2679-2688, 2005.

27. He H, Zang LH, Feng YS, et al: Physalin A induces apoptotic cell death and protective autophagy in HT1080 human fibrosarcoma cells. J Nat Prod 76: 880-888, 2013.

28. Mizushima N, Levine B, Cuervo AM and Klionsky DJ: Autophagy fights disease through cellular self-digestion. Nature 451: 1069-1075, 2008

29. Ye L, Zhao X, Lu J, Qian G, Zheng JC and Ge S: Knockdown of TIGAR by RNA interference induces apoptosis and autophagy in HepG2 hepatocellular carcinoma cells. Biochem Biophys Res Commun 437: 300-306, 2013.

30. Oh SY, Choi SJ, Kim KH, Cho EY, Kim JH and Roh CR: Autophagy-related proteins, LC3 and Beclin-1, in placentas from pregnancies complicated by preeclampsia. Reprod Sci 15: 912-920, 2008.

31. Mann SS and Hammarback JA: Molecular characterization of light chain 3. A microtubule binding subunit of MAP1A and MAP1B. J Biol Chem 269: 11492-11497, 1994.

32. Lang T, Schaeffeler E, Bernreuther D, Bredschneider M, Wolf DH and Thumm M: Aut2p and Aut7p, two novel microtubule-associated proteins are essential for delivery of autophagic vesicles to the vacuole. EMBO J 17: 3597-3607, 1998.

33. Kabeya Y, Mizushima N, Yamamoto A, Oshitani-Okamoto S, Ohsumi Y and Yoshimori T: LC3, GABARAP and GATE16 localize to autophagosomal membrane depending on form-II formation. J Cell Sci 117: 2805-2812, 2004.

34. Ying L, Huang Y, Chen H, et al: Downregulated MEG3 activates autophagy and increases cell proliferation in bladder cancer. Mol Biosyst 9: 407-411, 2013. 